Supporting information

\title{
Construction and characterization of a gradient strength promoter library for fine-tuned gene expression in Bacillus licheniformis
}

Yi Rao ${ }^{a}$, Peifen $\mathrm{Li}^{a}$, Xinxin Xie ${ }^{a}, \mathrm{Jiemin}_{\mathrm{Li}^{a}}$, Yongqing Liao ${ }^{a}, \mathrm{Xin} \mathrm{Ma}^{a}$, Dongbo Cai ${ }^{*}$,

Shouwen Chen ${ }^{a, b^{*}}$

a State Key Laboratory of Biocatalysis and Enzyme Engineering, Environmental Microbial

Technology Center of Hubei Province, College of Life Sciences, Hubei University, Wuhan, 430062,

PR China

${ }^{b}$ Fujian Provincial Key Laboratory of Eco-Industrial Green Technology, College of Ecological and Resource Engineering, Wuyi University, Wuyishan 354300, PR China

${ }^{*}$ Corresponding author: Prof. Shouwen Chen and Dr. Dongbo Cai

Tel./fax.: +86 027-88666081.

E-mail address: me1212@126.com (S. Chen) and caidongbo@hubu.edu.cn (D. Cai).

Postal address: 368 Youyi Avenue, Wuchang District, Wuhan 430062, Hubei, PR China 
Table S1 Strains and plasmids used in this study

\begin{tabular}{|c|c|c|}
\hline Strains & Characteristics & Source \\
\hline \multicolumn{3}{|l|}{ Escherichia coli } \\
\hline DH5 $\alpha$ & $\begin{array}{c}\mathrm{F}^{-} \Phi 80 \mathrm{~d} / \text { lacZ } \Delta \mathrm{M} 15, \Delta(\text { lacZYA-argF }) \mathrm{U} 169, \text { recA1, endA } 1, \\
\text { hsdR17 }\left(r_{\mathrm{K}^{-}}, m_{\mathrm{K}}{ }^{+}\right), \text {phoA, supE44, } \lambda^{-}, \text {thi-1, gyrA96, relA } 1\end{array}$ & Stored in this lab \\
\hline $5 \alpha / 300$ & DH5 $\alpha$ contains plasmid pHY300PLK & This study \\
\hline $5 \alpha / \mathrm{pP}_{\text {Ubay }}-\mathrm{GFP}$ & DH5 $\alpha$ contains plasmid $\mathrm{pHY}-\mathrm{P}_{\mathrm{Ubay}}-\mathrm{GFP}$ & This study \\
\hline $5 \alpha / \mathrm{pP}_{\mathrm{bacA}}-\mathrm{GFP}$ & DH5 $\alpha$ contains plasmid $\mathrm{pHY}-\mathrm{P}_{\mathrm{bac} A}-\mathrm{GFP}$ & This study \\
\hline $5 \alpha / \mathrm{pP}_{\text {Undh }}-\mathrm{GFP}$ & DH5 $\alpha$ contains plasmid $\mathrm{pHY}-\mathrm{P}_{\mathrm{Undh}}-\mathrm{GFP}$ & This study \\
\hline $5 \alpha /$ pP $P_{\text {Ubay- }}$ RFP & DH5 $\alpha$ contains plasmid $\mathrm{pHY}-\mathrm{P}_{\text {Ubay }}-\mathrm{RFP}$ & This study \\
\hline $5 \alpha / \mathrm{pP}_{\mathrm{bacA}}-\mathrm{RFP}$ & DH5 $\alpha$ contains plasmid $\mathrm{pHY}-\mathrm{P}_{\mathrm{bacA}}-\mathrm{RFP}$ & This study \\
\hline $5 \alpha / \mathrm{pP}_{\mathrm{Undh}}-\mathrm{RFP}$ & DH5 $\alpha$ contains plasmid $\mathrm{pHY}-\mathrm{P}_{\text {Undh }}-\mathrm{RFP}$ & This study \\
\hline \multicolumn{3}{|l|}{ Bacillus licheniformis } \\
\hline DW2 & Wide-type & Stored in lab \\
\hline DWc9n* & $\begin{array}{l}\text { B.licheniformis DW2 derivative with Cas9n integrated } \\
\text { expression and bacABC operon knocked out }\end{array}$ & Stored in lab \\
\hline DW2/300 & DW2 contains plasmid pHY300PLK & This study \\
\hline $\mathrm{DW} 2 / \mathrm{pP}_{\mathrm{bacA}}-\mathrm{GFP}$ & DW2 contains plasmid $\mathrm{pHY}-\mathrm{P}_{\mathrm{bacA}}-\mathrm{GFP}$ & This study \\
\hline $\mathrm{DW} 2 / \mathrm{pP}_{\mathrm{ylB}}-\mathrm{GFP}$ & DW2 contains plasmid $\mathrm{pHY}-\mathrm{P}_{\mathrm{ylB}}-\mathrm{GFP}$ & This study \\
\hline $\mathrm{DW} 2 / \mathrm{pP} \mathrm{P}_{\text {Ubay }}-\mathrm{GFP}$ & DW2 contains plasmid $\mathrm{pHY}-\mathrm{P}_{\mathrm{Ubay}}-\mathrm{GFP}$ & This study \\
\hline $\mathrm{DW} 2 / \mathrm{pP}_{\mathrm{UdhbF}}-\mathrm{GFP}$ & DW2 contains plasmid $\mathrm{pHY}-\mathrm{P}_{\mathrm{UdhbF}}-\mathrm{GFP}$ & This study \\
\hline $\mathrm{DW} 2 / \mathrm{pP}_{\mathrm{UgapA}}-\mathrm{GFP}$ & DW2 contains plasmid pHY-P $\mathrm{PgapA}_{\mathrm{A}}-\mathrm{GFP}$ & This study \\
\hline $\mathrm{DW} 2 / \mathrm{pP}_{\text {UyoaJ }}-\mathrm{GFP}$ & DW2 contains plasmid pHY-P $\mathrm{P}_{\text {Uyoa }}-\mathrm{GFP}$ & This study \\
\hline $\mathrm{DW} 2 / \mathrm{pP}_{\mathrm{UmalA}}$-GFP & DW2 contains plasmid $\mathrm{pHY}-\mathrm{P}_{\mathrm{UmalA}}$-GFP & This study \\
\hline $\mathrm{DW} 2 / \mathrm{pP}_{\mathrm{UropC}}-\mathrm{GFP}$ & DW2 contains plasmid $\mathrm{pHY}-\mathrm{P}_{\mathrm{UropC}}-\mathrm{GFP}$ & This study \\
\hline $\mathrm{DW} 2 / \mathrm{pP}_{\mathrm{UyvgO}}-\mathrm{GFP}$ & DW2 contains plasmid $\mathrm{pHY}-\mathrm{P}_{\mathrm{UyvgO}}-\mathrm{GFP}$ & This study \\
\hline $\mathrm{DW} 2 / \mathrm{pP}_{\mathrm{UbacC}}-\mathrm{GFP}$ & DW2 contains plasmid pHY-P $\mathrm{P}_{\mathrm{Ubac}}-\mathrm{GFP}$ & This study \\
\hline $\mathrm{DW} 2 / \mathrm{pP}_{\mathrm{UcspB}}-\mathrm{GFP}$ & DW2 contains plasmid pHY-P $\mathrm{UcspB}-\mathrm{GFP}$ & This study \\
\hline $\mathrm{DW} 2 / \mathrm{pP}_{\mathrm{UatpD}}-\mathrm{GFP}$ & DW2 contains plasmid $\mathrm{pHY}-\mathrm{P}_{\text {UatpD-GFP }}$ & This study \\
\hline $\mathrm{DW} 2 / \mathrm{pP}_{\mathrm{UmetH}^{-}}-\mathrm{GFP}$ & DW2 contains plasmid $\mathrm{pHY}-\mathrm{P}_{\mathrm{Umet}^{-}}-\mathrm{GFP}$ & This study \\
\hline $\mathrm{DW} 2 / \mathrm{pP}_{\mathrm{UtyrA}}-\mathrm{GFP}$ & DW2 contains plasmid pHY-P $\mathrm{PtyrA}_{\mathrm{U}} \mathrm{GFP}$ & This study \\
\hline $\mathrm{DW} 2 / \mathrm{pP}_{\mathrm{Umdh}}-\mathrm{GFP}$ & DW2 contains plasmidp pHY-P ${ }_{U m d h}-G F P$ & This study \\
\hline $\mathrm{DW} 2 / \mathrm{pP}_{\mathrm{UmdxF}}-\mathrm{GFP}$ & DW2 contains plasmidp pHY-P $\mathrm{P}_{\mathrm{UmdxF}}-\mathrm{GFP}$ & This study \\
\hline $\mathrm{DW} 2 / \mathrm{pP} \mathrm{Undh}_{\mathrm{h}}-\mathrm{GFP}$ & DW2 contains plasmid pHY-P ${ }_{\text {Undh }}-\mathrm{GFP}$ & This study \\
\hline DW2/pP43-GFP & DW2 contains plasmid pHY-P43-GFP & This study \\
\hline $\mathrm{DW} 2 / \mathrm{pP}_{\text {Ubay }}-\mathrm{RFP}$ & DW2 contains plasmid $\mathrm{pHY}-\mathrm{P}_{\mathrm{Ubay}}-\mathrm{RFP}$ & This study \\
\hline $\mathrm{DW} 2 / \mathrm{pP}_{\mathrm{bacA}}-\mathrm{RFP}$ & DW2 contains plasmid $\mathrm{pHY}-\mathrm{P}_{\mathrm{bacA}}-\mathrm{RFP}$ & This study \\
\hline $\mathrm{DW} 2 / \mathrm{pP} \mathrm{Undh}_{-\mathrm{RFP}}$ & DW2 contains plasmid $\mathrm{pHY}-\mathrm{P}_{\text {Undh }}-\mathrm{RFP}$ & This study \\
\hline $\mathrm{DW} 2 / \mathrm{pP}_{\text {Ubay-}} \mathrm{KER}$ & DW2 contains plasmid pHY-P ${ }_{\text {Ubay }}-K E R$ & This study \\
\hline $\mathrm{DW} 2 / \mathrm{pP}_{\mathrm{bacA}}-\mathrm{KER}$ & DW2 contains plasmid pHY-P $\mathrm{bacA}_{\mathrm{A}}-\mathrm{KER}$ & This study \\
\hline
\end{tabular}




\begin{tabular}{|c|c|c|}
\hline \multirow[b]{2}{*}{$\mathrm{DW} 2 / \mathrm{pP}_{\mathrm{Undh}}-\mathrm{KER}$} & \multirow[b]{2}{*}{ DW2 contains plasmid $\mathrm{pHY}-\mathrm{P}_{\text {Undh }}-\mathrm{KER}$} & \multirow[b]{2}{*}{ This study } \\
\hline & & \\
\hline DW2-P $\mathrm{Pbay}_{(\mathrm{bac} A B C)}$ & Replace the original promoter $\mathrm{P}_{\text {bacA }}$ with $\mathrm{P}_{\text {Ubay }}$ in DW2 & This study \\
\hline DW2-P $\mathrm{Undh}_{\text {(bacABC) }}$ & Replace the original promoter $\mathrm{P}_{\text {bacA }}$ with $\mathrm{P}_{\mathrm{Undh}}$ in DW2 & This study \\
\hline DWc9n*-P ${ }_{\text {Ubay }}(l e u S)$ & DWc9n* derivative, with leuS under control of promoter $\mathrm{P}_{\text {Ubay }}$ & This study \\
\hline DWc9n*-P $\mathrm{UyygO}_{\text {(leuS })}$ & DWc9n* derivative, with leuS under control of promoter $\mathrm{P}_{\mathrm{UyvgO}}$ & This study \\
\hline DWc9n*-P $\mathrm{Undh}_{\mathrm{h}}(\mathrm{leuS})$ & DWc9n* derivative, with leuS under control of promoter $\mathrm{P}_{\mathrm{Undh}}$ & This study \\
\hline DWc9n*-P Ubay $(\mathrm{yvmC})$ & DWc9n* derivative, with $y v m C$ under control of promoter $\mathrm{P}_{\text {Ubay }}$ & This study \\
\hline DWc9n*-P $\left.\mathrm{UyvgO}_{\mathrm{yvmC}}\right)$ & $\begin{array}{l}\text { DWc9n* derivative, with } y v m C \text { under control of promoter } \\
\qquad \mathrm{P}_{\mathrm{UyvgO}}\end{array}$ & This study \\
\hline DWc9n*-P ${ }_{\text {Undh }}(\mathrm{yvmC})$ & DWc9n* derivative, with $y v m C$ under control of promoter $\mathrm{P}_{\text {Undh }}$ & This study \\
\hline PQM-1 & $\begin{array}{c}\text { DWc9n* derivative, with } b k d A B \text { under control of } \\
\text { promoter } \mathrm{P}_{\text {Undh }}\end{array}$ & This study \\
\hline PQM-2 & $\begin{array}{l}\text { PQM-1 derivative, with leuS under control of promoter } \\
\qquad \mathrm{P}_{\mathrm{UyvgO}}\end{array}$ & This study \\
\hline PQM-3 & $\begin{array}{l}\text { PQM-2 derivative, with } y v m C \text { under control of } \\
\text { promoter } \mathrm{P}_{\text {Ubay }}\end{array}$ & This study \\
\hline \multicolumn{3}{|l|}{ Plasmids } \\
\hline pHY300PLK & $\begin{array}{l}\text { E. coli-Bacillus shuttle vector; Ampr in E. coli, Tcr in } \\
\text { both E. coli and B. licheniformis }\end{array}$ & Stored in lab \\
\hline pGRNA & pHY300PLK+P43(no RBS)+gRNA+TamyL & Stored in lab \\
\hline T2(2)-Ori & Bacillus knockout vector; Kanr & Stored in lab \\
\hline 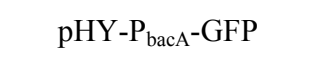 & pHY300PLK harbors $\mathrm{P}_{\text {bacA }}$-GFP-TamyL expression cassette & This study \\
\hline pHY-P ${ }_{y l B}-G F P$ & pHY300PLK harbors $\mathrm{P}_{\mathrm{ylB}}$-GFP-TamyL expression cassette & Stored in lab \\
\hline pHY-P ${ }_{\text {Ubay }}-\mathrm{GFP}$ & pHY300PLK harbors $\mathrm{P}_{\text {Ubay }}$-GFP-TamyL expression cassette & This study \\
\hline $\mathrm{pHY}-\mathrm{P}_{\mathrm{UdhbF}}-\mathrm{GFP}$ & pHY300PLK harbors $\mathrm{P}_{\mathrm{UdhbF}}$-GFP-TamyL expression cassette & This study \\
\hline pHY-P ${ }_{\text {UgapA }}-\mathrm{GFP}$ & pHY300PLK harbors $\mathrm{P}_{\text {UgapA-GFP-TamyL expression cassette }}$ & This study \\
\hline pHY-P ${ }_{\text {UyoaJ }}-G F P$ & pHY300PLK harbors $\mathrm{P}_{\mathrm{UyoaJ}}-\mathrm{GFP}-\mathrm{TamyL}$ expression cassette & This study \\
\hline $\mathrm{pHY}-\mathrm{P}_{\mathrm{UmalA}}-\mathrm{GFP}$ & pHY300PLK harbors $\mathrm{P}_{\text {UmalA-GFP-TamyL expression cassette }}$ & This study \\
\hline pHY-P ${ }_{\text {UropC }}-\mathrm{GFP}$ & pHY300PLK harbors $\mathrm{P}_{\mathrm{UropC}}$-GFP-TamyL expression cassette & This study \\
\hline pHY-P $\mathrm{UyvgO}-\mathrm{GFP}$ & pHY300PLK harbors $\mathrm{P}_{\mathrm{UyvgO}}$-GFP-TamyL expression cassette & This study \\
\hline pHY-P ${ }_{\text {UbacC }}-\mathrm{GFP}$ & pHY300PLK harbors $\mathrm{P}_{\mathrm{UbacC}}$-GFP-TamyL expression cassette & This study \\
\hline pHY-P ${ }_{U c s p B}-G F P$ & pHY300PLK harbors $\mathrm{P}_{\mathrm{Ucsp}}$-GFP-TamyL expression cassette & This study \\
\hline pHY-P ${ }_{\text {UatpD-GFP }}$ & pHY300PLK harbors $P_{\text {UatpD }}$-GFP-TamyL expression cassette & This study \\
\hline pHY-P ${ }_{U m e t H}-G F P$ & pHY300PLK harbors $\mathrm{P}_{\mathrm{UmetH}^{-} \mathrm{GFP}-\mathrm{TamyL} \text { expression cassette }}$ & This study \\
\hline pHY-P $\mathrm{UtyrA}_{\mathrm{A}}-\mathrm{GFP}$ & pHY300PLK harbors $P_{\text {UtyrA }}$-GFP-TamyL expression cassette & This study \\
\hline pHY-P $\mathrm{Umdh}-\mathrm{GFP}$ & pHY300PLK harbors $\mathrm{P}_{\mathrm{Umdh}}$-GFP-TamyL expression cassette & This study \\
\hline $\mathrm{pHY}-\mathrm{P}_{\mathrm{UmdxF}}-\mathrm{GFP}$ & pHY300PLK harbors $\mathrm{P}_{\mathrm{UmdxF}}$-GFP-TamyL expression cassette & This study \\
\hline pHY-P ${ }_{\text {Undh }}-\mathrm{GFP}$ & pHY300PLK harbors $\mathrm{P}_{\text {Undh }}$-GFP-TamyL expression cassette & This study \\
\hline pHY-P43-GFP & pHY300PLK harbors P43-GFP-TamyL expression cassette & Stored in lab \\
\hline pHY-P Ubay - RFP & pHY300PLK harbors $\mathrm{P}_{\text {Ubay }}-\mathrm{RFP}-\mathrm{TamyL}$ expression cassette & This study \\
\hline pHY-P ${ }_{\text {bacA }}-\mathrm{RFP}$ & pHY300PLK harbors $\mathrm{P}_{\text {bacA }}$-RFP-TamyL expression cassette & This study \\
\hline pHY-P $\mathrm{Pndh}_{\mathrm{Un}}-\mathrm{RFP}$ & pHY300PLK harbors $\mathrm{P}_{\text {Undh }}$-RFP-TamyL expression cassette & This study \\
\hline pHY-P $\mathrm{Pbay}_{\text {Ua }}-\mathrm{KER}$ & pHY300PLK harbors $\mathrm{P}_{\text {Ubay }}$-KER-TamyL expression cassette & This study \\
\hline
\end{tabular}




\begin{tabular}{|c|c|c|}
\hline pHY-P bacA-KER & pHY300PLK harbors $\mathrm{P}_{\text {bacA-KER-TamyL expression cassette }}$ & This study \\
\hline pHY-P ${ }_{\text {Undh-KER }}$ & pHY300PLK harbors $\mathrm{P}_{\text {Undh-KER-TamyL expression cassette }}$ & This study \\
\hline $\mathrm{T} 2-\mathrm{P}_{\text {Ubay }}(\mathrm{bacABC})$ & $\mathrm{T} 2(2)-$ Ori- $\mathrm{P}_{\text {Ubay }}\left(\mathrm{A}+\mathrm{P}_{\text {Ubay }}+\mathrm{B}\right)$, to replace the promoter of $\mathrm{P}_{\mathrm{bacA}}$ & This study \\
\hline T2-P $\mathrm{P}_{\text {Undh }}(\mathrm{bacABC})$ & $\begin{array}{l}\mathrm{T} 2 \text { (2)-Ori- } \mathrm{P}_{\text {Undh }}\left(\mathrm{A}+\mathrm{P}_{\mathrm{Undh}}+\mathrm{B}\right) \text {, to replace the promoter of } \\
\qquad \mathrm{P}_{\mathrm{bacA}}\end{array}$ & This study \\
\hline pPGRNA-P Ubay $($ leuS) & $\begin{array}{c}\mathrm{pHY} 300 \mathrm{PLK}+\mathrm{P} 43(\text { noRBS })+\mathrm{gRNA}\left(\mathrm{P}_{\text {leuS }}\right)+\mathrm{TamyL}+\mathrm{LH}+\mathrm{P}_{\text {Ubay }}+ \\
\mathrm{RH}\end{array}$ & This study \\
\hline pPGRNA-P & $\begin{aligned}\text { pHY300PLK }+ \text { P43(noRBS }) & + \text { gRNA }\left(\mathrm{P}_{\text {leus }}\right)+\text { TamyL }+\mathrm{LH}+\mathrm{P}_{\mathrm{UyvgO}} \\
+ & \mathrm{RH}\end{aligned}$ & This study \\
\hline pPGRNA-P ${ }_{\text {Undh }}($ leuS $)$ & $\begin{array}{c}\mathrm{pHY} 300 \mathrm{PLK}+\mathrm{P} 43(\mathrm{noRBS})+\mathrm{gRNA}\left(\mathrm{P}_{\text {leuS }}\right)+\mathrm{TamyL}+\mathrm{LH}+\mathrm{P}_{\text {Undh }}+ \\
\mathrm{RH}\end{array}$ & This study \\
\hline pPGRNA-P ${ }_{\text {Ubay }}(\mathrm{yvmC})$ & $\begin{aligned}\text { pHY300PLK }+ \text { P43(noRBS }) & +g R N A\left(P_{y v m C}\right)+\text { TamyL }+ \text { LH }+P_{\text {Ubay }} \\
& +\mathrm{RH}\end{aligned}$ & This study \\
\hline pPGRNA-P ${ }_{\text {Uyvgo }}(y v m C)$ & $\begin{aligned} & \mathrm{pHY} 300 \mathrm{PLK}+\mathrm{P} 43(\text { noRBS })+ \mathrm{gRNA}\left(\mathrm{P}_{\mathrm{yvmC}}\right)+\mathrm{TamyL}+\mathrm{LH}+\mathrm{P}_{\mathrm{UyvgO}} \\
&+\mathrm{RH}\end{aligned}$ & This study \\
\hline pPGRNA-P ${ }_{\text {Undh }}(y v m C)$ & $\begin{aligned} \mathrm{pHY} 300 \mathrm{PLK}+\mathrm{P} 43(\mathrm{noRBS}) & +\mathrm{gRNA}\left(\mathrm{P}_{\mathrm{yvmC}}\right)+\mathrm{TamyL}+\mathrm{LH}+\mathrm{P}_{\mathrm{Undh}} \\
& +\mathrm{RH}\end{aligned}$ & This study \\
\hline pPGRNA-P ${ }_{\text {Undh }}(\mathrm{bkdAB})$ & $\begin{array}{c}\mathrm{pHY} 300 \mathrm{PLK}+\mathrm{P} 43(\text { noRBS })+\mathrm{gRNA}\left(\mathrm{P}_{\mathrm{bkdAB}}\right)+\mathrm{TamyL}+\mathrm{LH}+\mathrm{P}_{\text {Undh }} \\
+\mathrm{RH}\end{array}$ & This study \\
\hline
\end{tabular}


Table S2 Primers used in this study

\begin{tabular}{|c|c|}
\hline Primers & Sequence of primer (5' to $3^{\prime}$ ) \\
\hline $300-\mathrm{TF}$ & GAATTCCTGTTATAAAAAAAGGA \\
\hline 300-GR & ATGGTGAGCAAGGGCGAGGAGCTG \\
\hline $\mathrm{P}_{\text {bacA }}-\mathrm{F}$ & TTTTTATAACAGGAATTCCCTGCGATTTCGGCGAGATTC \\
\hline $\mathrm{P}_{\mathrm{bacA}}-\mathrm{R}$ & CTCGCCCTTGCTCACCATATAAAAATTCTCCTTTTTG \\
\hline $\mathrm{P}_{\text {Ubay }}-\mathrm{F}$ & ATCTCCCCCTTTGTTGTTTCATTCACCATAATATTGGTC \\
\hline $\mathrm{P}_{\text {Ubay }}-\mathrm{R}$ & CAACAAAGGGGGAGATTTGTATGGTGAGCAAGGGCGAGGAG \\
\hline $\mathrm{P}_{\text {UdhbF-F }}$ & CATTGATAGTCTGCATTTGGCAAGGGTGCTTCTATTCACCATAATATTGGTC \\
\hline $\mathrm{P}_{\mathrm{UdhbF}}-\mathrm{R}$ & AAATGCAGACTATCAATGAAGGAGGTCGCCTTAATGGTGAGCAAGGGCGAGG \\
\hline $\mathrm{P}_{\text {UgapA }}-\mathrm{F}$ & TTTCCTCCTTTAAGTAAATAAGAATTCACCATAATATTGGTC \\
\hline $\mathrm{P}_{\text {UgapA }}-\mathrm{R}$ & TTTACTTAAAGGAGGAAATCATCATGGTGAGCAAGGGCGAGG \\
\hline $\mathrm{P}_{\text {Uyoas }}-\mathrm{F}$ & CCTCCTGTTTTATAAATTGAGAACATTCACCATAATATTGGTC \\
\hline $\mathrm{P}_{\text {Uyoa }}-\mathrm{R}$ & AATTTATAAAACAGGAGGATGGATATGGTGAGCAAGGGCGAGG \\
\hline $\mathrm{P}_{\text {UmalA }}-\mathrm{F}$ & AAAGCCCCCTTATAAGCGTTTAATTCACCATAATATTGGTC \\
\hline $\mathrm{P}_{\text {UmalA }}-\mathrm{R}$ & CGCTTATAAGGGGGCTTTAAAAATGGTGAGCAAGGGCGAGG \\
\hline $\mathrm{P}_{\text {UropC }}-\mathrm{F}$ & GGGCCTACCTCCСТTTTTAATTCACCATAATATTGGTC \\
\hline$P_{\text {Uropc }}-\mathrm{R}$ & AAAAAGGGAGGTAGGCCCCATGGTGAGCAAGGGCGAGG \\
\hline $\mathrm{P}_{\text {Uyvgo- }} \mathrm{F}$ & GATTTACCCTCCTAAAATAAATTCACCATAATATTGGTC \\
\hline $\mathrm{P}_{\text {Uyvgo }}-\mathrm{R}$ & TTATTTTAGGAGGGTAAATCATGGTGAGCAAGGGCGAGG \\
\hline $\mathrm{P}_{\text {Ubacc }}-\mathrm{F}$ & $\begin{array}{l}\text { TTCCTTTTGTTTTCAGATCCACATGGGCCGCCCATTTTTTATTCACCATAATATTGGT } \\
\text { C }\end{array}$ \\
\hline $\mathrm{P}_{\text {Ubacc }}-\mathrm{R}$ & $\begin{array}{l}\text { ATCTGAAAACAAAAGGAATTCTAGGAGATGAGATGACGATATGGTGAGCAAGGGC } \\
\text { GAGG }\end{array}$ \\
\hline $\mathrm{P}_{\mathrm{UcspB}}-\mathrm{F}$ & TCCTCCTAAAGCGATAACGCTTAAATTCACCATAATATTGGTC \\
\hline$P_{\text {UcspB }}-R$ & GTTATCGCTTTAGGAGGAAATTTCATGGTGAGCAAGGGCGAGG \\
\hline $\mathrm{P}_{\text {UatpD }}-\mathrm{F}$ & CTCTATCCCTCCTGACAAGCATTCACCATAATATTGGTC \\
\hline $\mathrm{P}_{\text {UatpD }}-\mathrm{R}$ & GCTTGTCAGGAGGGATAGAGATGGTGAGCAAGGGCGAGG \\
\hline $\mathrm{P}_{\mathrm{UmetH}}-\mathrm{F}$ & TTCTTTCTGGTGGATATATGCCGTCAGTTCGGATTCACCATAATATTGGTC \\
\hline $\mathrm{P}_{\text {UmetH }}-\mathrm{R}$ & ATATATCCACCAGAAAGAAAAGGAGAAAACAAATGGTGAGCAAGGGCGAGG \\
\hline $\mathrm{P}_{\mathrm{UtyrA}}-\mathrm{F}$ & CCTAAGTTCACTAAAGAGGTGACTGAAATTCACCATAATATTGGTC \\
\hline $\mathrm{P}_{\mathrm{UtyrA}}-\mathrm{R}$ & CTCTTTAGTGAACTTAGGTGATAAAAAATGGTGAGCAAGGGCGAGG \\
\hline $\mathrm{P}_{\text {Umdh }}-\mathrm{F}$ & GTTTTCTACСССТTTTAATATTCACCATAATATTGGTC \\
\hline $\mathrm{P}_{\text {Umdh }}-\mathrm{R}$ & ATTAAAAGGGGTAGAAAACATGGTGAGCAAGGGCGAGG \\
\hline $\mathrm{P}_{\text {UmdxF }}-\mathrm{F}$ & СCTCTATTCTTCCGGACGCTAAAACCGATTCACCATAATATTGGTC \\
\hline $\mathrm{P}_{\mathrm{UmdxF}}-\mathrm{R}$ & CGTCCGGAAGAATAGAGGGCGAAAAAAATGGTGAGCAAGGGCGAGG \\
\hline
\end{tabular}




\begin{tabular}{|c|c|}
\hline $\mathrm{P}_{\text {Undh }}-\mathrm{F}$ & ATCCTCCGTCCTTATTCTGAATGATTCACCATAATATTGGTC \\
\hline $\mathrm{P}_{\text {Undh }}-\mathrm{R}$ & AGAATAAGGACGGAGGATTCACGATGGTGAGCAAGGGCGAGG \\
\hline Ubay-F & ACAAАTCTCCCССТTTGTTG \\
\hline bacA-F & ATAAAАATTCTCCTTTTTGAT \\
\hline Undh-F & CGTGAATCCTCCGTCCTTATTC \\
\hline T5-R & AAGAGCAGAGAGGACGGATTTCC \\
\hline Ubay-KER-F & ACAAAGGGGGAGATTTGTATGAGAGGCAAAAAGGTATG \\
\hline bacA-KER-F & AAAAAGGAGAATTTTTATATGAGAGGCAAAAAGGTATG \\
\hline Undh-KER-F & AAGGACGGAGGATTCACGATGAGAGGCAAAAAGGTATG \\
\hline KER-R & TCCGTCCTCTCTGCTCTTTTACTGAGCTGCCGCCTGTAC \\
\hline Ubay-RFP-F & CAACAAAGGGGGAGATTTGTATGGTTTCCAAAGGAGAAGAA \\
\hline bacA-RFP-F & TTCTCCTTTGGAAACCATATAAAAATTCTCCTTTTTG \\
\hline Undh-RFP-F & AGAATAAGGACGGAGGATTCACGATGGTTTCCAAAGGAGAAGAA \\
\hline RFP-R & ATGGTTTCCAAAGGAGAAGAA \\
\hline BAU-YF & GCTCATCAATCATGAAGAGC \\
\hline BAU-YR & ACTGTAGTGCGGGGTGATG \\
\hline BAUbay-F1 & GCTCTAGAGATTGCGACATTACCGTTC \\
\hline BAUbay-R1 & СTCCCCСTTTGTTGTTTCATTCACCATAATATTGGTC \\
\hline BAUbay-F2 & GAAACAACAAAGGGGGAGATTTGTATGGTTGCTAAACATTCATTAG \\
\hline BAUbay-R2 & CCGAGCTCGTCTGAAGCCTCTCTGTCC \\
\hline BUndh-F & ATCCTCCGTCCTTATTCTGAATGATTCACCATAATATTGGTC \\
\hline BUndh-R & AGAATAAGGACGGAGGATTCACGATGGTTGCTAAACATTCATTAG \\
\hline pHY-F & GTTTATTATCCATACCCTTAC \\
\hline pHY-R & CAGATTTCGTGATGCTTGTC \\
\hline bkd-g-F & AGTGATAGCGGTACCATTACCGAACCGCTTTTTTATACGTTTTAGAGCTAGAAATAG \\
\hline bkd-g-R & TAATGATGACGGTCCAGC \\
\hline bkd-F1 & GCTGGACCGTCATCATTAGCGAAGACTTGTACTACC \\
\hline bkd-R1 & TCTCGCCGAAATCGCAGGTTATTGCATGCTATCTTTTGC \\
\hline bkd-F2 & CCTGCGATTTCGGCGAGATTC \\
\hline bkd-R2 & TAATTGTTTCAGCTTCATCGTGAATCCTCCGTCCTT \\
\hline bkd-F3 & ATGAAGCTGAAACAATTATTG \\
\hline bkd-R3 & TTTGCCCAAGCTTCTAGAAAGCGGCTTTCGGCATTTGC \\
\hline
\end{tabular}




\begin{tabular}{|c|c|}
\hline bkd-YF & AAGCGCTCGCTGAGGGCA \\
\hline UbayL-F & AATCTCCCCCTTTGTTGTTTCATTCACCATAATATTGGTC \\
\hline UbayL-R & ACAACAAAGGGGGAGATTTGTTTGAGTTTTGACCATCAAAC \\
\hline Ubay-kF & GAAACAACAAAGGGGGAG \\
\hline Ubay-kR & CAAGAACGGTTCCAAGCG \\
\hline UndhL-F & ATCCTCCGTCCTTATTCTGAATGATTCACCATAATATTGGTC \\
\hline UndhL-R & AGAATAAGGACGGAGGATTCACGTTGAGTTTTGACCATCAAAC \\
\hline UndhL-KF & AAGGACGGAGGATTCACG \\
\hline UyvgOL-F & ATTTACCCTCCTAAAATAAATTCACCATAATATTGGTC \\
\hline UyvgOL-R & TATTTTAGGAGGGTAAATCTTGAGTTTTGACCATCAAAC \\
\hline UyvgOY-F & GATTTACCCTCCTAAAATAAATTCACCATAATATTGGTC \\
\hline UyvgOY-R & TTATTTTAGGAGGGTAAATCATGACAGAGCTTACAATGG \\
\hline UyvgO-KF & ATTTTAGGAGGGTAAATC \\
\hline UyvgO-KR & TGGACATCCATCCGAATG \\
\hline UbayY-F & AATCTCCCCCTTTGTTGTTTCATTCACCATAATATTGGTC \\
\hline UbayY-R & ACAACAAAGGGGGAGATTTGTATGACAGAGCTTACAATGG \\
\hline UndhY-F & ATCCTCCGTCCTTATTCTGAATGATTCACCATAATATTGGTC \\
\hline UndhY-R & AGAATAAGGACGGAGGATTCACGATGACAGAGCTTACAATGG \\
\hline RT-GFP-F & GGTGAACTTCAAGATCCGCC \\
\hline RT-GFP-R & CTTGTACAGCTCGTCCATGC \\
\hline RT-16s-F & ACCTAACCAGAAAGCCACGG \\
\hline RT-16s-R & GTTTACGGCGTGGACTACCA \\
\hline GFP-blot-F & TGAGCAAGGGCGAGGAGCTGT \\
\hline GFP-blot-R & TAATACGACTCACTATAGGATGCTTGTCGGCCATGATATAGA \\
\hline 16s-blot-F & CTGGTCTGTAACTGACGCTGAG \\
\hline 16s-blot-R & TAATACGACTCACTATAGGAACCCAACATCTCACGACAC \\
\hline
\end{tabular}


Table S3 Transcriptome data of different promoters used in this study

\begin{tabular}{cc}
\hline Promoters & Transcriptome data in B. licheniformis DW2 \\
\hline $\mathbf{P}_{\text {bacC }}$ & 728297 \\
$\mathbf{P}_{\text {bacA }}$ & 712366 \\
$\mathbf{P}_{\text {dhbF }}$ & 411591 \\
$\mathbf{P}_{\text {yvgO }}$ & 195196 \\
$\mathbf{P}_{\text {malA }}$ & 126056 \\
$\mathbf{P}_{\text {cspB }}$ & 65903 \\
$\mathbf{P}_{\text {rpoC }}$ & 56055 \\
$\mathbf{P}_{\text {yoaJ }}$ & 49279 \\
$\mathbf{P}_{\text {atpD }}$ & 38480 \\
$\mathbf{P}_{\text {gapA }}$ & 27132 \\
$\mathbf{P}_{\text {mdh }}$ & 26152 \\
$\mathbf{P}_{\text {mdxF }}$ & 25517 \\
$\mathbf{P}_{\text {tyrA }}$ & 15786 \\
$\mathbf{P}_{\text {ndh }}$ & 11549 \\
$\mathbf{P}_{\text {metH }}$ & 9604 \\
\hline
\end{tabular}


Table S4 5' -UTR sequences of different promoters used in this study

\begin{tabular}{cc}
\hline promoters & 5' -UTR sequence \\
\hline $\mathrm{P}_{\text {bacA }}$ & ATTTTATCAAAAAGGAGAATTTTTAT \\
$\mathrm{P}_{\text {ylB }}$ & GAAACAACAAAGGGGGAGATTTGT \\
$\mathrm{P}_{\text {Ubay }}$ & GAAACAACAAAGGGGGAGATTTGT \\
$\mathrm{P}_{\text {UdhbF }}$ & AGAAGCACCCTTGCCAAATGCAGACTATCAATGAAGGAGGTCGCCTTA \\
$\mathrm{P}_{\text {UgapA }}$ & TCTTATTTACTTAAAGGAGGAAATCATC \\
$\mathrm{P}_{\text {Uyoal }}$ & GTTCTCAATTTATAAAACAGGAGGATGGAT \\
$\mathrm{P}_{\text {UmalA }}$ & TAAACGCTTATAAGGGGGCTTTAAAA \\
$\mathrm{P}_{\text {UropC }}$ & TAAAAAGGGAGGTAGGCCCC \\
$\mathrm{P}_{\text {Uyvgo }}$ & TTATTTTAGGAGGGTAAATC \\
$\mathrm{P}_{\text {Ubacc }}$ & AAAAAATGGGCGGCCCATGTGGATCTGAAAACAAAAGGAATTCTAGGAGATGAGAT \\
$\mathrm{P}_{\text {UcspB }}$ & TTAAGCGTTATCGCTTTAGGAGGAAATTTC \\
$\mathrm{P}_{\text {UatpD }}$ & GCTTGTCAGGAGGGATAGAG \\
$\mathrm{P}_{\text {UmetH }}$ & CCGAACTGACGGCATATATCCACCAGAAAGAAAAGGAGAAAACAA \\
$\mathrm{P}_{\text {UtyrA }}$ & TTCAGTCACCTCTTTAGTGAACTTAGGTGATAAAAA \\
$\mathrm{P}_{\text {Umdh }}$ & ATTAAAAGGGGTAGAAAAC \\
$\mathrm{P}_{\text {UmdxF }}$ & CATTCAGAATAAGGACGGAGGATTCACG \\
$\mathrm{P}_{\text {Undh }}$ &
\end{tabular}


Figure S1 The 5' -UTR secondary structure map of 17 promoters

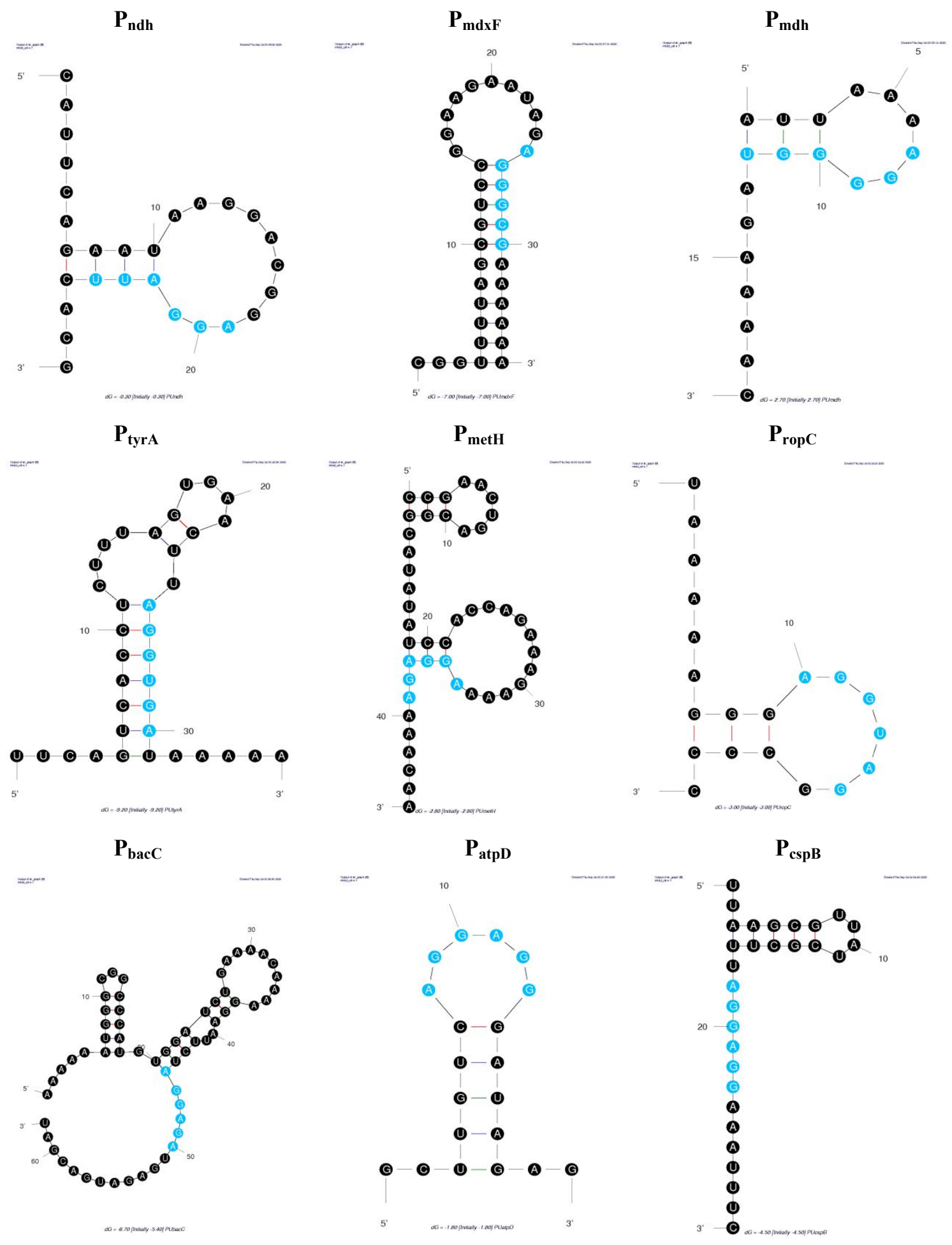




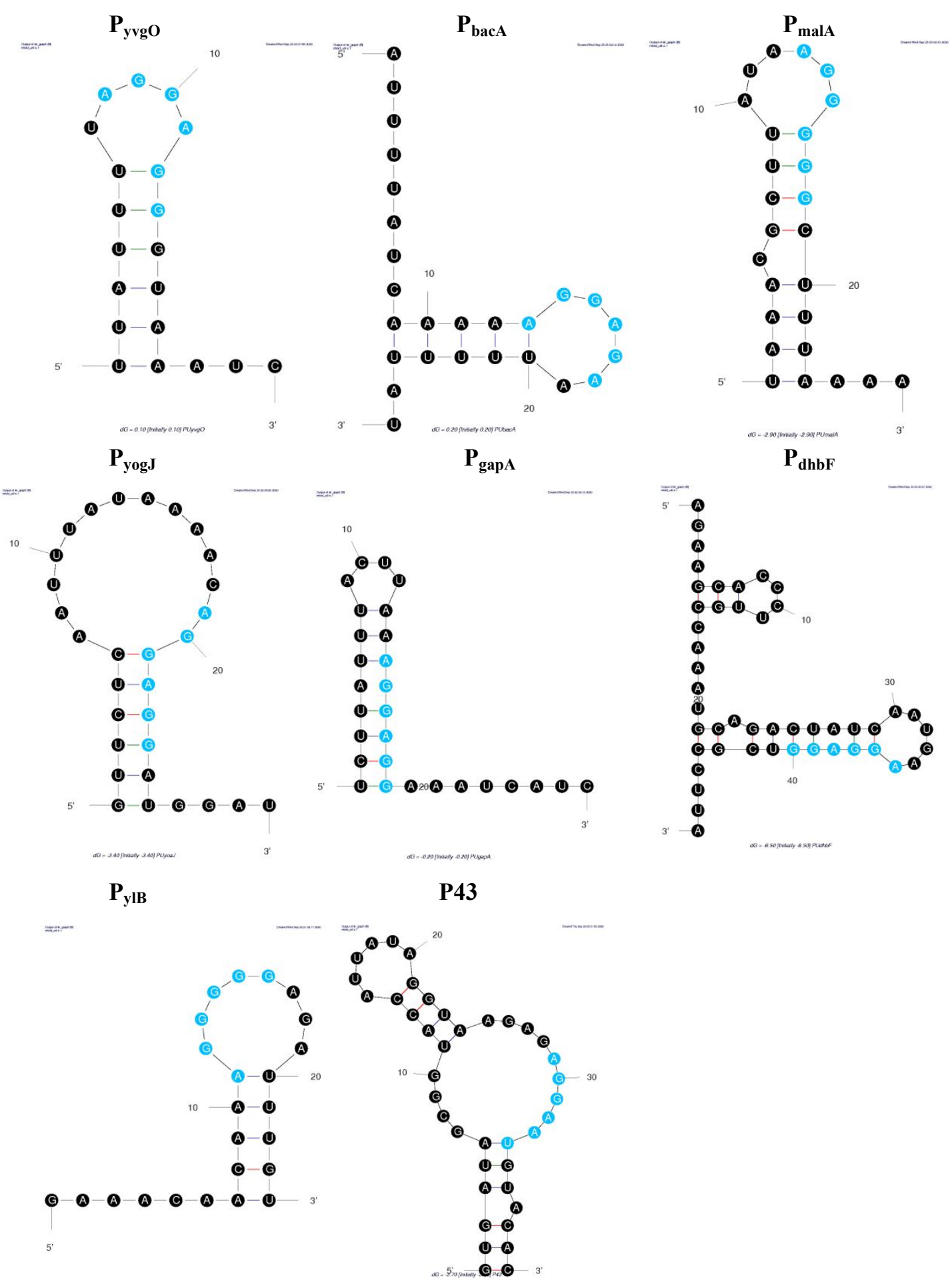

Notes: Blue is SD sequence 
Figure S2 The cell biomass of 17 different recombinant strains at $24 \mathrm{~h}$

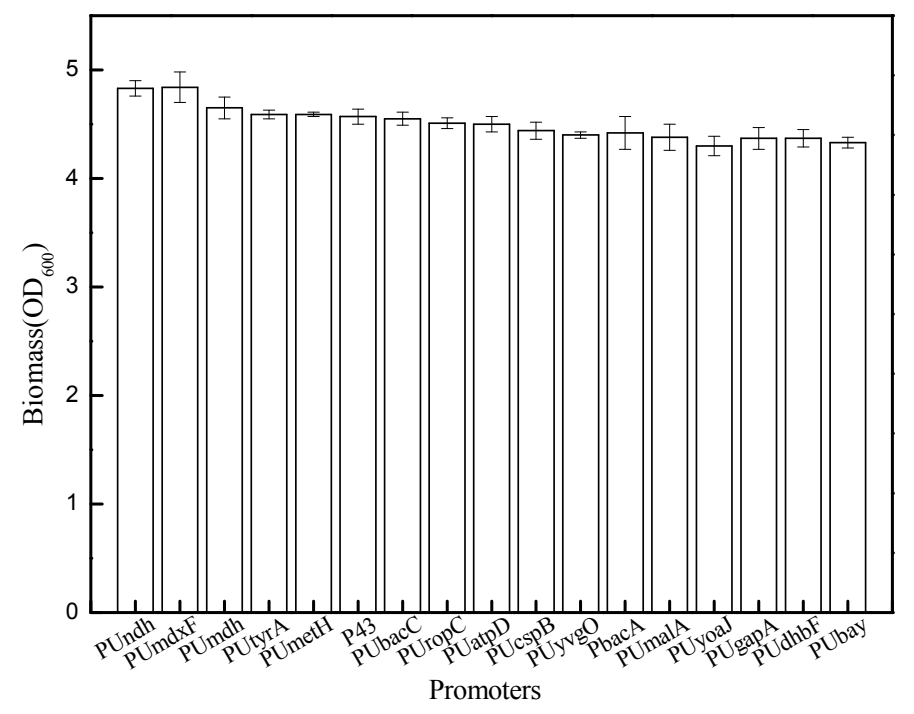


Figure S3 The strength of promoters ( $\mathrm{P} 43, \mathrm{P}_{\mathrm{bacA}}$ and $\left.\mathrm{P}_{\mathrm{Ubay}}\right)$ in $B$. subtilis 168 and $B$. licheniformis DW2.

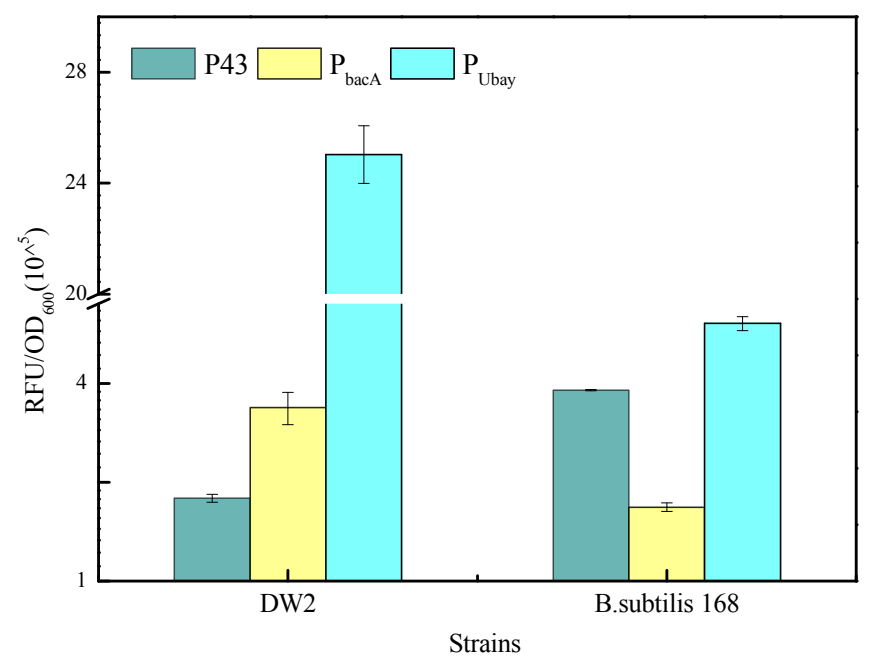


Figure S4 Correlation analysis of transcription levels to 5'-UTR performance at 17 different promoters.

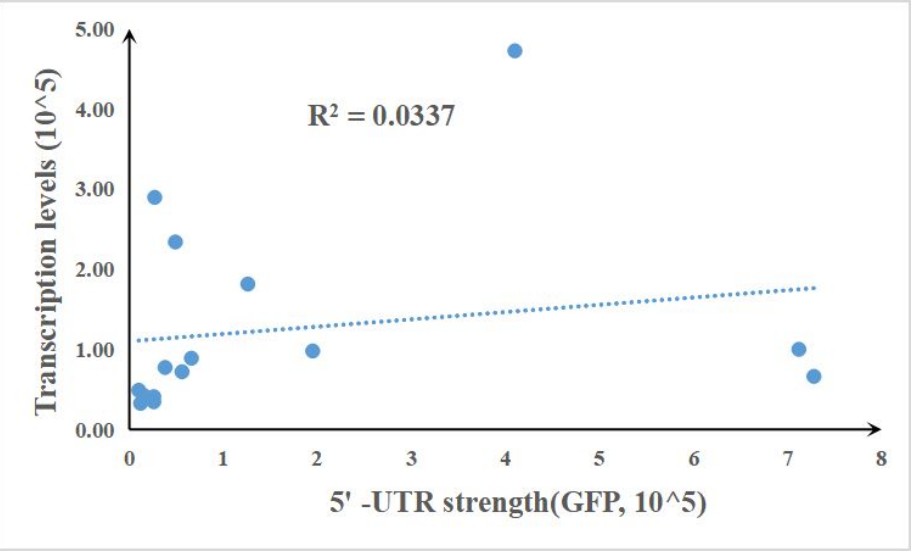


Figure S5 The secondary structure map of 5' -UTR with the first 30 bp of gene gfp of 17 promoters
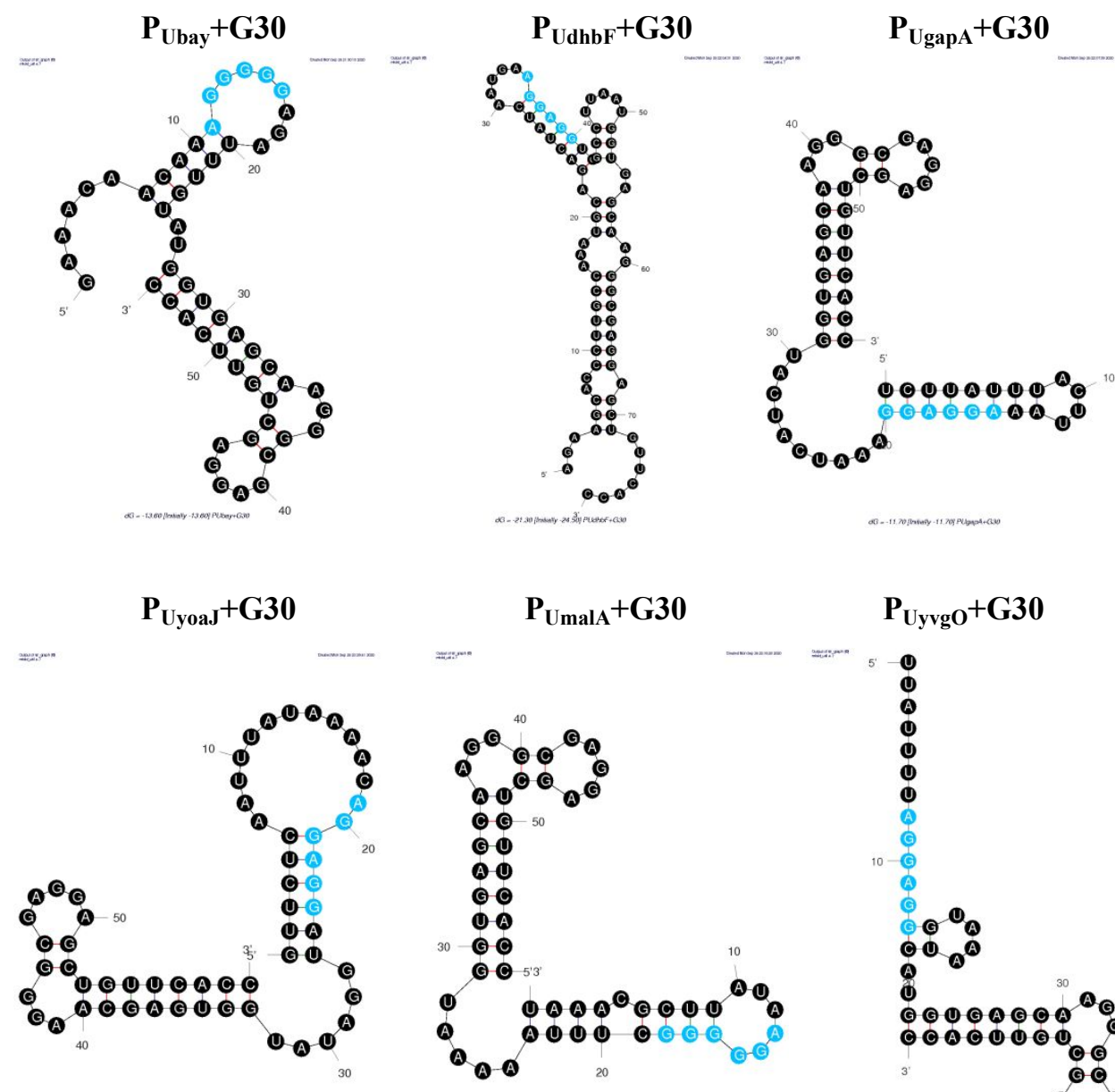

$$
\mathbf{P}_{\mathrm{UyvgO}}+\mathbf{G 3 0}
$$
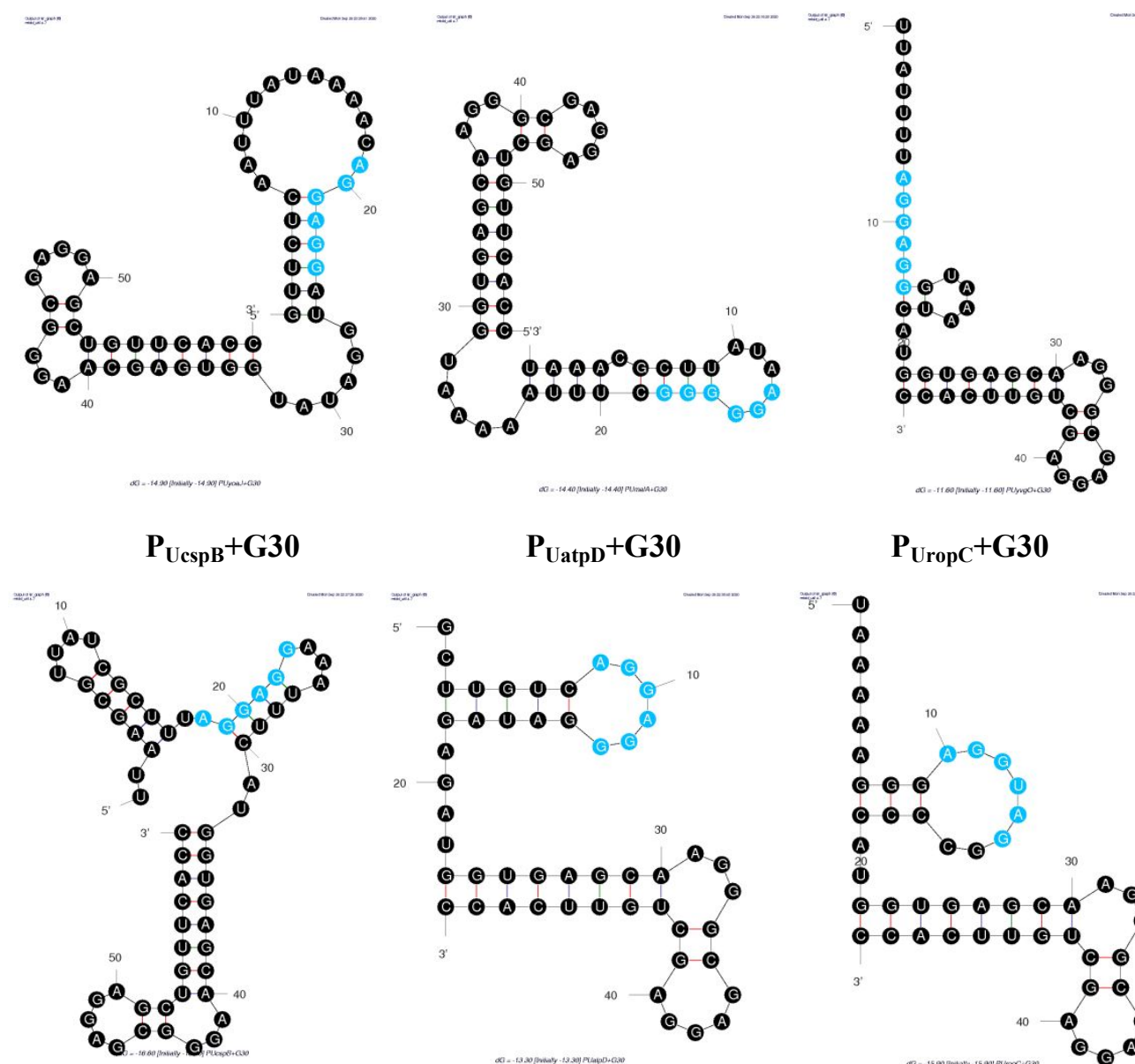

$$
\text { P }_{\text {UropC }}+\mathbf{G 3 0}
$$

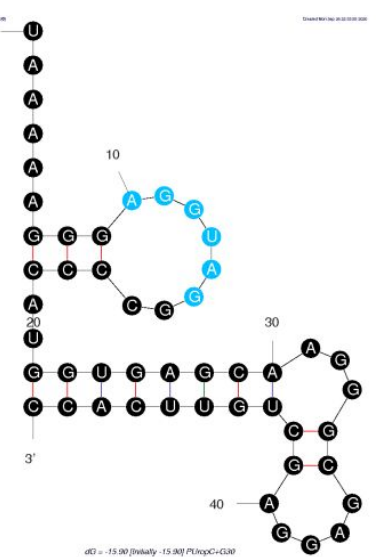




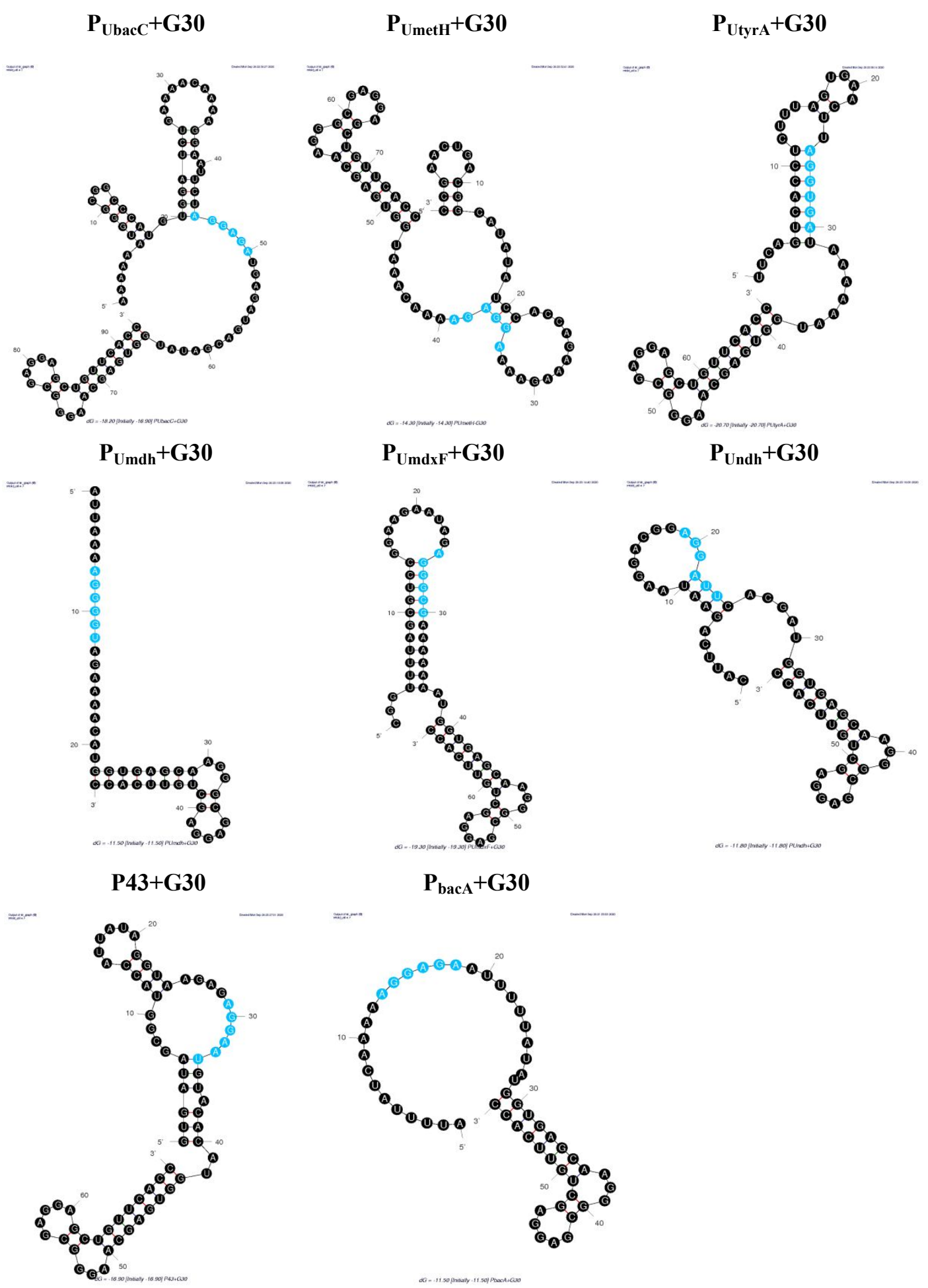

Notes: Blue is SD sequence 
Figure S6 Effect of the length of the N-terminal sequence of $g f p$ gene on the secondary structure of 5'-UTR.

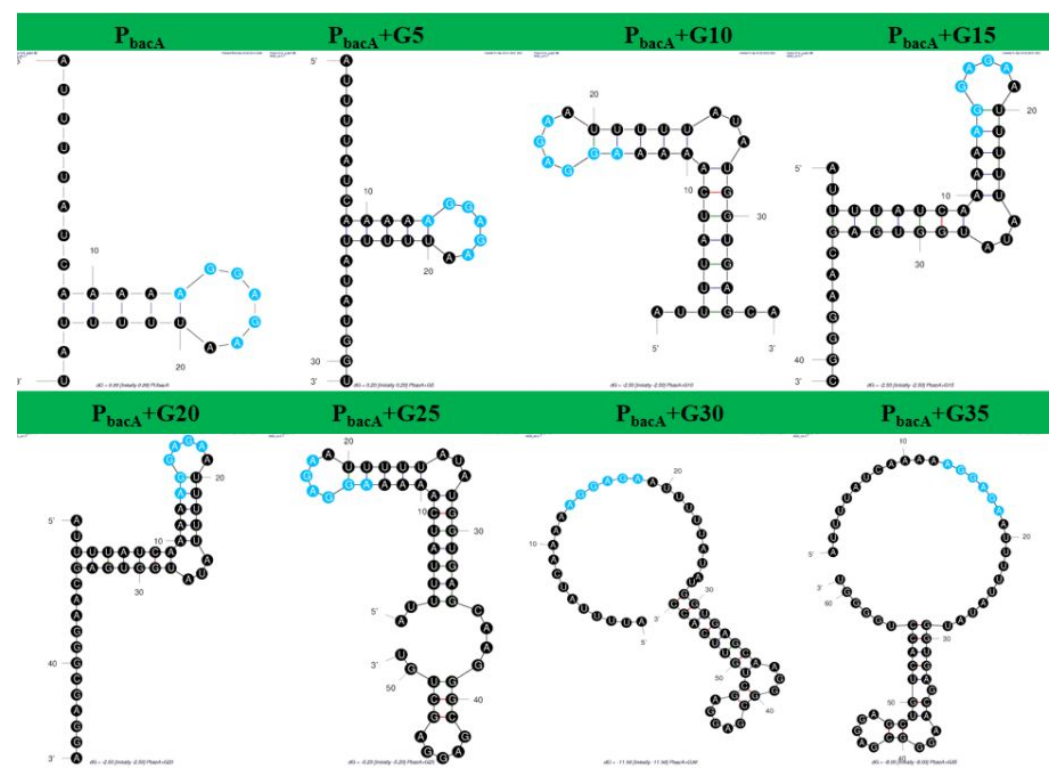

